\title{
Enfoque cualimétrico: Necesidad cultural para el desarrollo universitario
}

\author{
Scientific research with quealimetric approach: A cultural need \\ for university development
}

Recibido: agosto 10 de 2017 | Revisado: octubre 18 de 2017 | Aceptado: noviembre 16 de 2017

George Argota Pérez

RubÉN Ticona Huayhua ${ }^{2}$

Grover Marín Mamaní ${ }^{3}$

Francisco Curro Pérez ${ }^{3}$

Rosalvina Campos Pérez ${ }^{4}$
1 Centro de Investigaciones Avanzadas y formación Superior en Educación, Salud y Medio Ambiente "AMTAWI". Puno - Perú. george.argota@gmail.com

2 Universidad Nacional del Altiplano. Puno - Perú gscaspatig@usmp.pe

3 Universidad Nacional de Juliaca. Puno - Perú gmarin@unaj.edu.pe f.curro@unaj.edu.pe.

4 Universidad Nacional Federico Villarreal. Lima - Perú rosalvina17@gmail.com

\section{RESUMEN}

En este trabajo, hemos considerado que la implementación de la investigación científica con enfoque cualimétrico es una necesidad cultural para el desarrollo universitario, contrario a lo que existe actualmente en universidades públicas y privadas que se basan en un formato de investigación con una estructura metodológica, por lo general, cuantitativa. En ese sentido, considerar el estado de opinión sobre los actores involucrados en un fenómeno, suceso, evento o proceso donde no necesariamente, tendría que iniciarse demostrando determinada hipótesis. Por tanto, la razón cognoscitiva tendría que estar concebida desde el entendimiento y dominio de los silogismos deductivo e inductivo para enfrentar un enfoque cualimétrico al iniciar cualquier tipo de investigación científica.

Palabras clave: investigación cualimétrica, cultura científica, desarrollo universitario

\section{Abstract}

In this research, we have considered that the implementation of scientific research with a qualimetric approach is a cultural necessity for university development, contrary to what currently exists in public and private universities, which are based on a research format with a methodological structure, usually, quantitative. In this sense, considering the state of opinion on the actors involved in a phenomenon, event or process where, not necessarily, it would have to start by demonstrating a certain hypothesis. Therefore, the cognitive reason should be conceived from the understanding and mastery of the deductive and inductive syllogisms to face a qualimetric approach when initiating any type of scientific research.

Key words: qualimetric research, scientific culture, university development 


\section{Introducción}

La contribución universitaria para la sociedad, independientemente del país al que pertenezca, constituye una tendencia cada vez más creciente (Kosmützky \& Krücken, 2014; Lane, 2011) donde existen diversos criterios o esfuerzos para clasificar las actividades que las universidades realizan (Kinser \& Lane, 2015). Uno de estos criterios se refiere al potencial de investigación (Pfotenhauer, Wood, Roos \& Newman, 2016) donde existen disímiles acciones y/o actuaciones para realizarlas como pueden ser a través del propio contexto relativo al campus donde se ubican o mediante su relación en un país extranjero (Li et al., 2016; Shapira, Youtie \& Taylor, 2016). Hernández, Fernández y Baptista (2010) señalan que la característica esencial y naturaleza misma de la universidad moderna es la investigación científica y formación de recurso humano altamente calificado. Sin embargo, una de las principales causas que está limitando la solución en diversos problemas de hacer investigación científica, radica en identificar los efectos negativos considerando un enfoque $u$ otro debido a la formación profesional, y así se entiende por los propios formatos de perfiles y proyectos de tesis de investigación presentados en las universidades (Argota \& Iannacone, 2015).

Artigas y Robles (2010) afirman que es necesario educar a los investigadores en relación con la combinación de los enfoques cualitativo y cuantitativo para que el seguimiento con respecto a determinado esquema (tipo de investigación), no imposibilite las innovaciones en la búsqueda sobre el mejoramiento del proceso de investigación, además, de no evidenciar falta de conocimiento a la hora de construir un determinado silogismo. En este sentido, los tipos de investigación representan modelos de conducción que informan el tipo de investigación a realizarse, no siempre, resultan comprensibles en cuanto a las informaciones consideradas. Diversos tipos de investigación, en las universidades, son divergentes quizás justificados por la propia autonomía universitaria (reconocida en el Perú a través de la Ley $N^{\circ} 30220$ ), pues cada universidad considera que lo propuesto es, justificadamente, lo más correcto, aunque lo criticable radica en que la inmensa mayoría de los formatos señalan en estructura dinámica, un enfoque por lo general, cuantitativo. En ese sentido, el propósito de nuestro trabajo fue considerar la implementación sobre la investigación científica con enfoque cualimétrico como necesidad cultural para el desarrollo universitario del Perú.

\section{Desarrollo}

Barletta, et al (2017) mencionan que en los últimos años, existe un especial interés sobre el análisis en la gestión del conocimiento como factor determinante de la innovación, siendo esta perspectiva muy diferente en cada uno de los países (Chaves et al., 2015; García, De Castro Araújo, Mascarino \& Dos Santos, 2011; Rapini, Chiarini \& Bittencourt, 2015). Al mencionarse innovación, es razonable que se pueda pensar que solo haya sido producto de una conjetura del estado de opinión de una población destinataria para reconocer los tipos de impactos a ofrecer. Esta reflexión permite reconocer que la formación en la praxis científica universitaria, no puede estar direccionada a un tipo de enfoque, aunque parezca ser lo contrario. 
La ciencia tiene una como principal característica, la objetividad (Argota, 2017). Reconocer que existe una forma única hacia un tipo de estructura metodológica, que es la valoración fundamental en este artículo. Colmenares, Mercedes, Piñero y Lourdes (2008) señalan que, en los escenarios de la investigación científica, a través de los siglos, se han generado grandes cambios que marcan diferencias y mejoras significativas en la manera del cómo abordar el objeto de estudio y donde al revisarse la evolución de la propia investigación, la misma se encuentra dividida en etapas bien delimitadas por dos grandes perspectivas o enfoques que, a su vez, han conducido al desarrollo de diferentes tendencias o metodologías que permiten incursionar en el campo de la investigación por diferentes vías. Los propios autores indican que estas dos tendencias de investigación albergan un abanico de alternativas metodológicas, pero al parecer la más usual ha sido con base en términos cuantitativos como se observa en diversas universidades. Puede interpretarse que el silogismo inductivo que caracteriza la relación sujeto-actor(es) involucrado(s) en el contexto de la observación, tendría menor significación y ello sin duda es un error, ya que pensar que los métodos cualitativos por fenomenología, etnografía, etnometodología, teoría fundamentada, investigación acción y biografía presentan menos criterios científicos en el tratamiento de ideas que los métodos teóricos, empíricos y de enfoque que caracterizan al silogismo deductivo (cuantitativo) de investigación (Argota \& Iannacone, 2015; Cornejo, Mendoza \& Rojas, 2008).
Weinberg (1999) advierte, en su reflexión sobre el método científico que no sirve para tener ideas, por el contrario, hay que tratarlas de manera que, en las investigaciones con enfoques cualitativos, las percepciones de los investigadores sobre los actores involucrados en el contexto del problema social, igualmente conseguirán tratarse y al conocerse que no se debe seguir un modelo rígido indicaría, asimismo, la validez del conocimiento adquirido en comparación con la demostración de hipótesis que caracteriza la investigación cuantitativa (Cornejo \& Salas, 2011). El mismo Weinberg (1999) señaló que la historia sobre la ciencia, es aquella de las buenas preguntas y por consiguiente, es la de buenas respuestas lo que reconoce el gran significado y valor de la investigación cualitativa conjuntamente con la cuantitativa, siendo corroborado por Feynman (1999) quien expresó que, el método científico alude más a la forma de tratar las ideas que a su propio origen. Asimismo, Christ (2007) argumentó que la investigación mediante métodos mixtos se ha fortalecido en los últimos veinte años y los estudios exploratorios cualitativos seguidos de estudios confirmatorios han sido comunes y concurrentes, con lo cual se sigue demostrando que, solo enfrentar investigaciones sobre una determinada posición, quizás podría mitigar el problema científico. Cameron (2009) indicó que la investigación con métodos mixtos ha ido generando cambios metodológicos entre los investigadores y académicos en una variedad de disciplinas por cuanto, el dominio de cada silogismo, resulta esencial para transformar los efectos negativos (Figura 1). 


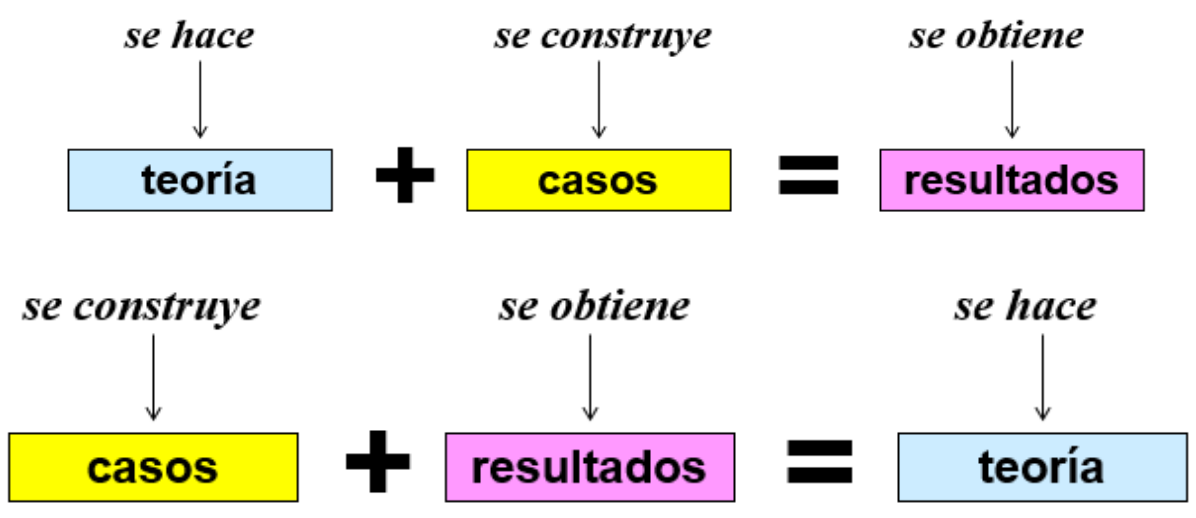

Figura 1. Silogismos de investigación (superior: deductivo, inferior: inductivo) Fuente: Enríquez y Argota (2016)

Un ejemplo sencillo, en el área ambiental, puede ilustrar que la falta de dominio sobre los silogismos, conduce a incorrectas toma de decisiones. El 10 de marzo del año 2013, en la ciudad de Puno, se observó la muerte masiva de peces y la población se preguntó: ¿qué habia ocurrido? Ante tal acontecimiento, diversas instituciones dedicadas a la investigación científica, realizaron mediciones in-situ y a nivel de laboratorio. Aunque, se informó técnicamente condiciones anómalas para la vida acuática, solo ello representó suposiciones. Sin embargo, al escucharse, paralelamente, el estado de opinión en los pescadores de la zona (Chimú) se valoraron algunos detalles que, finalmente, fueron los que contribuyeron a explicar el posible suceso, pues algunas dudas con relación al debate científico, no permitieron converger en la problemática. No obstante, la información de los pescadores como actores involucrados posibilitó la aproximación de ideas para entender las explicaciones científicas que ellos expresaron. En este ejemplo, no fue la investigación de tipo cuantitativa de forma inicial, lo que justificó el hecho, sino la investigación cualitativa, a través del mé- todo por fenomenología; y esto demuestra, la necesidad de no parametrarse en un determinado tipo de investigación.

Finalmente, si bien la investigación científica resulta imprescindible para toda aquella persona que pretenda identificar, plantear, estudiar y solucionar, eficientemente, las necesidades y problemas de la sociedad, también debe entenderse que como proceso mediante la aplicación del método científico procura obtener información relevante y fidedigna para entender, verificar, corregir o aplicar un determinado conocimiento y actividad que está caracterizada por ser reflexiva, sistemática y metódica, ya sea por vía deductiva como inductiva o viceversa (Argota, 2016).

\section{Conclusiones}

La razón cognoscitiva frente a cualquier estado diferente al deseado, está concebida desde el entendimiento y dominio de los silogismos deductivo e inductivo y solo así, es posible enfrentar un enfoque cualimétrico al iniciar cualquier tipo de investigación científica. 


\section{Referencias}

Argota, P.G. (2016). Razonamiento lógico e interpretación práctica del perfil y proyecto tesis de investigación cientifica: pensamiento con enfoque cualimétrico. Cusco, Perú: Editorial KOPYgraf.

Argota, P.G. (2017). Glosario terminológico: análisis ambiental y metodología de la investigación cientifica. Cusco, Perú: Editorial KOPYgraf.

Argota, P.G. \& Iannacone, O.J. (2015). Significación de formatos metodológicos como guías de aprendizaje para la investigación científica de postgrado en ciencias naturales y no afines de universidades peruanas. Revista The Biologist; 13(1), 41-51. doi:http://dxdoi.org/10.24039/ rtb201513141

Artigas, W. \& Robles, M. (2010). Metodología de la investigación: Una discusión necesaria en Universidades. Revista Digital Universitaria, 11, 16. Recuperado de http:// www.revista.unam.mx/vol.11/ num11/art107/art107.pdf

Barletta, F., Yoguela, G., Pereira, M. \& Rodríguez, S. (2017). Exploring scientific productivity and transfer activities: Evidence from Argentinean ICT research groups. Research Policy, 46(8), 1-9. doi:http://dx.doi. org/10.1016/j.respol.2017.05.007

Cameron, R. (2009). The use of mixed methods research in VET research, Proceedings of aligning participants policy and pedagogy: traction and tension. In VET research: 12th AVETRA Annual Conference, Sydney, NSW, 15-17, AVETRA, Crows Nest. Recuperado de http://epubs.scu.edu.au/cgi/viewcontent.cgi?article $=1$ 158\&contex$\mathrm{t}=$ comm_pubs

Chaves, C.V., Rapini, M.S., Suzigan, W., Fernandes, A.C., Domingues, E. \& Carvalho, S.S. (2015). The contribution of universities and research institutes to the Brazilian innovation system. Innov. Dev., 5, 1-20. doi:http://dx.doi.org/10.1080/215 7930X.2015.1056401

Christ, T. (2007). A Recursive Approach to Mixed Methods Research in a Longitudinal Study of Postsecondary Education Disability Support Services. Journal of Mixed Methods Research, 1(3), 226-241.

Colmenares, E., Mercedes, A., Piñero, M. \& Lourdes, M. (2008). La investigación acción. Una herramienta metodológica heurística para la comprensión y transformación de realidades y prácticas socio-educativas. Revista Laurus, 14, 96-114. Recuperado de http://www.redalyc.org/articulo. oa?id=76111892006

Cornejo, M. \& Salas, N. (2011). Rigor y calidad metodológicos: un reto a la investigación social cualitativa. Psicoperspectivas. Revista Individuo y Sociedad, 10(2), 12-34. Recuperado de http://www.scielo.cl/pdf/ psicop/v10n2/art02.pdf

Cornejo, M., Mendoza, F. \& Rojas, R.C. (2008). La investigación con relatos de vida: pistas y opciones del diseño metodológico. Revista Psykhe, 17, 2939. Recuperado de http://www.scielo.cl/pdf/psykhe/v17n1/art04.pdf 
Enríquez, S.P. \& Argota, P.G. (2016). Descripción interpretativa para la elaboración del perfil de tesis de investigación científica con enfoque cualimétrico (mixto). Revista CAMPUS, 22(22), 151-164. doi.org/10.24265/ campus.2016.v21n 22.02

Feynman, RP. (1999). Qué significa todo eso: Reflexiones de un cientifico ciudadano. Barcelona, España: Editorial Crítica.

García, R., De Castro Araújo, V., Mascarini, S. \& Dos Santos, E.G. (2011). Os efeitos da proximidade geográfica para o estímulo da interação universidade-empresa. Revista de Economia, 37, 307-330. doi: http://dx.doi.org/10.5380/re. v37i4.27687

Hernández, R., Fernández, C. \& Baptista, P. (2010). El proceso de investigación y los enfoques cuantitativos y cualitativos hacia un modelo integral. En (Eds.), Metodología de la investigación (pp. 2-16). España: McGraw-Hill Interamericana.

Kinser, K. \& Lane, J. (2015). Foreign outposts of colleges and universities. Int. High. Educ; 66, 2-3. doi:http://dx.doi.org/10.6017/ ihe.2012.66.8583

Kosmützky, A. \& Krücken, G. (2014). Macro-environmental Mapping of International Branch Campus Activities of Universities Worldwide. Research \& Occasional Paper Series, CSHE.2.14. Center for Studies in Higher Education. University of California, Berkeley. Recuperado de https://eric.ed.gov l?id=ED545192.
Lane, J. (2011). Importing private higher education: international branch campuses. J. Comp. Policy Anal.: Res. Pract. 13(4), 367-381. doi:http://dx.doi.org/10.1080/138 76988.2011.583106

Li, Y., Kolesnikov, S., Woo, S., Schimid, J., Adebola, O., Taylor, Z., ... Rogers, J. (2016). International University Research Ventures (IURV): Findings from U.S. Universities. STIP Working Paper. Georgia Tech Program in Science, Technology and Innovation Policy, Georgia Institute of Technology, Atlanta. doi:http://dx.doi.org/10.13140/ RG.2.2.35999.87204

Pfotenhauer, S.M., Wood, D., Roos, D. \& Newman, D. (2016). Architecting complex international science, technology and innovation partnerships (CISTIPs): a study of four global MIT collaborations. Technol. Forecast. Soc. Change, 104, 38-56. doi:http://dx.doi.org/10.1016/j. techfore.2015.12.006

Rapini, M.S., Chiarini, T. \& Bittencourt, P.F. (2015). University-firm interactions in Brazil: beyond human resources and training missions. Ind. High. Educ, 29(2), 111-127.

Shapira, P., Youtie, Y. \& Taylor, M.Z. (2016). What are the implications of the growth of international university research ventures? Paper Presented at the R \& D Management Conference. July 3-6, Cambridge, UK

Weinberg, S. (1999). Una visión corrosiva del progreso científico. Mundo Cientifico, 201, 74-82. 